Check for updates

Cite this: RSC Chem. Biol., 2021, 2, 1263

Received 31st May 2021,

Accepted 7th July 2021

DOI: 10.1039/d1cb00123j

rsc.li/rsc-chembio

\section{In vivo active organometallic-containing antimycotic agents $\dagger$}

\author{
Riccardo Rubbiani, ${ }^{\star a}$ Tobias Weil, (D)*b Noemi Tocci, (DD ${ }^{\mathrm{b}}$ Luciano Mastrobuoni, ${ }^{\mathrm{a}}$ \\ Severin Jeger, ${ }^{a}$ Marco Moretto, (D) ${ }^{c}$ James Ng, (D) ${ }^{d}$ Yan Lin, (D) d Jeannine Hess, (D) a \\ Stefano Ferrari, (D) ${ }^{e}$ Andres Kaech, ${ }^{f}$ Luke Young, (D) ${ }^{g}$ John Spencer, (D) 9 \\ Anthony L. Moore, (D) ${ }^{\mathrm{h}}$ Kevin Cariou, (D) *d Giorgia Renga, (D) Marilena Pariano, \\ Luigina Romani (D) ${ }^{i}$ and Gilles Gasser (D) *d
}

\begin{abstract}
Fungal infections represent a global problem, notably for immunocompromised patients in hospital, COVID-19 patient wards and care home settings, and the ever-increasing emergence of multidrug resistant fungal strains is a sword of Damocles hanging over many healthcare systems. Azoles represent the mainstay of antifungal drugs, and their mode of action involves the binding mode of these molecules to the fungal lanosterol $14 \alpha$-demethylase target enzyme. In this study, we have prepared and characterized four novel organometallic derivatives of the frontline antifungal drug fluconazole (1a-4a). Very importantly, enzyme inhibition and chemogenomic profiling demonstrated that lanosterol $14 \alpha$-demethylase, as for fluconazole, was the main target of the most active compound of the series, $(\mathrm{N}$-(ferrocenylmethyl)-2-(2,4-difluorophenyl)-2-hydroxy- $N$-methyl-3-(1H-1,2,4-triazol-1-yl)propan-1-aminium chloride, 2a). Transmission electron microscopy (TEM) studies suggested that $\mathbf{2} \mathbf{a}$ induced a loss in cell wall integrity as well as intracellular features ascribable to late apoptosis or necrosis. The impressive activity of $\mathbf{2 a}$ was further confirmed on clinical isolates, where antimycotic potency up to 400 times higher than fluconazole was observed. Also, $\mathbf{2 a}$ showed activity towards azole-resistant strains. This finding is very interesting since the primary target of $\mathbf{2 a}$ is the same as that of fluconazole, emphasizing the role played by the organometallic moiety. In vivo experiments in a mice model of Candida infections revealed that 2a reduced the fungal growth and dissemination but also ameliorated immunopathology, a finding suggesting that $\mathbf{2} \mathbf{a}$ is active in vivo with added activity on the host innate immune response.
\end{abstract}

\section{Introduction}

${ }^{a}$ Department of Chemistry, University of Zurich, Winterthurerstrasse 190, 8057 Zurich, Switzerland. E-mail: rubbianiric@gmail.com

${ }^{b}$ Department of Food Quality and Nutrition, Research and Innovation Centre, Fondazione Edmund Mach, Via E. Mach 1, 38010 San Michele all'Adige, Italy. E-mail: tobias.weil@fmach.it

${ }^{c}$ Unit of Computational Biology, Research and Innovation Centre, Fondazione Edmund Mach, Via E. Mach 1, 38010 San Michele all'Adige, Italy

${ }^{d}$ Chimie ParisTech, PSL University, CNRS, Institute of Chemistry for Life and Health Sciences, Laboratory for Inorganic Chemical Biology, 75005 Paris, France. E-mail: kevin.cariou@chimieparistech.psl.eu, gilles.gasser@chimieparistech.psl.eu

${ }^{e}$ Institute of Molecular Cancer Research, University of Zurich, Winterthurerstrasse 190, 8057 Zurich, Switzerland

${ }^{f}$ Center for Microscopy and Image Analysis, University of Zurich, Winterthurerstrasse 190, 8057 Zurich, Switzerland

${ }^{g}$ Department of Chemistry, School of Life Sciences, University of Sussex, Brighton BN1 9QJ, UK

${ }^{h}$ Biochemistry \& Biomedicine, School of Life Sciences, University of Sussex, Brighton $B N 1$ 9QG, UK

${ }^{i}$ University of Perugia, Department of Medicine and Surgery, Piazzale Lucio Severi - Polo Unico Sant'Andrea delle Fratte, 06132 Perugia, Italy

$\dagger$ Electronic supplementary information (ESI) available. See DOI: 10.1039/d1cb00123j
Mycoses are one of the most common opportunistic infections worldwide, affecting poor as well as industrialized countries. Skin infections (e.g. athlete's foot), for example, affect $20-25 \%$ of the world's population and systemic opportunistic infections are the 4th common cause of bloodstream infections with a lifetime incidence of about $75 \%$ (e.g. in the case of Candidiasis). ${ }^{1-6}$ Even if such extensive morbidity does not lead to a high mortality rate, local opportunistic infections together with invasive fungal infections (IFI) have a deep impact on the entire health system because they suppress the immune system and thus, increase the risk to develop other/additional pathologies, which can be fatal to immunosuppressed people. ${ }^{7-16}$ Although potent drugs (e.g. clotrimazole) are applied in antifungal therapy, the infection rate is worryingly increasing every year. Moreover, incidences of resistance to conventional antimycotics are steadily increasing, especially for long term therapies that last from several weeks to months. ${ }^{17}$ Main mechanisms necessary for antifungal resistance are drug target mutations (e.g. mutations of CYP51A1), 
upregulation of efflux channels (e.g. major facilitator super-family (MFS) and ATP-binding cassette (ABC channels)), aneuploidy and loss of heterozygosity $(\mathrm{LOH})$ and modulation of the stress response (e.g. translational signal pathways, RNA dependent response). ${ }^{18-21}$ Surprisingly, despite a pressing need for novel antifungal agent research and drug development, there is an apparent downturn in such activities. ${ }^{22}$ However, the recent COVID-19 pandemic has witnessed numerous examples of COVID-19 and C. auris co-infections, adding further pressure to healthcare systems. ${ }^{23}$

Cell wall targeting is at the basis of modern antifungal therapy since it is absent in mammalian cells allowing for selectivity. A selective destabilization or inhibition of cell wall biosynthesis leads to metabolic onset, growth arrest and culminates in fungal death. For decades, Amphotericin B deoxycholate (AmB) has been the only available treatment for invasive fungal infections (IFIs). ${ }^{24}$ However, during the last ten years, the growing number of IFI incidences has stimulated the introduction of four new classes of antifungal agents, namely polyene-based compounds, azoles, allylamines and echinocandins. ${ }^{25-28}$ Inhibition of cell wall biosynthesis is associated with an accumulation of sterol toxic precursors, cell wall destabilization and cellular stress. During the second half of the 20th century, antifungal research mainly focused on an intensive derivatization of accepted compounds (e.g. from ketoconazole to fluconazole of from AmB to Nystatin and AmB liposomes). ${ }^{19-21}$ Despite these efforts, to date, only seven compounds are included in the World Health Organization Essential Medicine list (WHO-EM, data of October 2013). ${ }^{1-6}$ Moreover, the benchmark drugs were all discovered almost 20 or more years ago and accounts of ineffective therapies have been reported for several of these agents especially towards Candida albicans (C. albicans) and Aspergillus terreus. ${ }^{1-6}$ For this reason, and encouraged by the spectacular results obtained with metal complexes (e.g. salvarsan, cisplatin, auranofin, ferroquine, ferrocifen, etc.) in other medicinal fields, ${ }^{29-38}$ some groups have decided to assess the potential of such compounds to fight fungal infections. ${ }^{39,40}$ Despite such a wealth of promising examples of metal-based drugs, there is a paucity of examples of such compounds applied in the field of antifungal therapy. Here, just sporadic reports confirmed the effectiveness of different transition metal complexes (e.g. $\mathrm{Co}(\mathrm{II}), \mathrm{Ni}(\mathrm{II}), \mathrm{Cu}(\mathrm{II}), \mathrm{Pd}(\mathrm{II})$ complexes) bearing a variety of ligands (e.g. azole moieties, thiosemicarbazones, carboxamides, indoles) against several fungal strains or some organometallic moieties bound to active antifungal agents. ${ }^{41-53}$ However, all these studies generally provided only $\mathrm{MIC}_{50}$ values without any further biological investigation such as target engagement and toxicity. Willing to grasp this opportunity, we embarked on a research program to unveil potent complexes against fungal infections with an emphasis on addressing cellular targets and mode of action. Herein, we present the identification of a metallocene-based lead compound against such infections.

\section{Results and discussion}

\section{Synthesis and characterization}

The two most common antifungal drugs inscribed in the List of Essential Medicine of the WHO and commercially available, namely clotrimazole and fluconazole (Fig. 1), belong to the azole class. The molecular target for both those azoles was found to be the enzyme lanosterol-14 $\alpha$-demethylase. ${ }^{47}$ of utmost interest, mammalian cells use cholesterol instead of ergosterol. This important difference secures high selectivity for the reported antimycotics. ${ }^{54}$ Recently, the broad therapeutic applications of clotrimazole as well as fluconazole against skin, genital and invasive fungal infections have generated comparison of primary or cross resistance, especially for Candidiasis. ${ }^{55,56}$ Motivated by the promising results obtained from the ferrocenyl derivatization of organic drugs such as tamoxifen and chloroquine to give ferrocifen and ferroquine, respectively, we envisaged applying the same concept to fight fungal infections. ${ }^{32,49,57-59}$ Fluconazole has been chosen as the parent drug because of its broad spectrum applications (e.g. systemic and topic administration, active against a large number of mycoses). The drug design took into consideration the mode of action of fluconazole and its interaction with the active site of the target enzyme. Computing binding geometry data showed how fluconazole activity is exerted by the interaction of the triazole moiety (i.e. the nitrogen atom highlighted in blue in Fig. 1) with the haem group present in the active site of the target enzyme (i.e. the iron atom). ${ }^{60}$ This interaction is also favoured by the presence of the difluorophenyl group that is located in the enzyme active site, in (close) proximity to the hydrophobic binding cleft and interacts with it via $\pi-\pi$

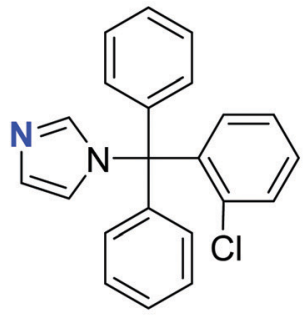

Clotrimazole<smiles>OC(Cn1cncn1)(Cn1cncn1)c1ccc(F)cc1F</smiles>

Fluconazole<smiles>[R]N(Cc1ccccc1)CC(O)(Cn1cncn1)c1ccc(F)cc1F</smiles>

$\begin{array}{ll}\mathrm{R}: \mathrm{H} & =\mathbf{1} \\ \mathrm{R}: \text { Methyl } & =\mathbf{2} \\ \mathrm{R}: \text { Ethyl } & =\mathbf{3} \\ \mathrm{R}: \text { Isopropyl } & =4\end{array}$

Fig. 1 Chemical structures of the main antifungal therapeutics present on the market (clotrimazole and fluconazole) and our fluconazenes 1-4, highlighted in blue are the nitrogen responsible for the binding with the haem group. 
stacking: ${ }^{60}$ On the contrary, the second triazole moiety is involved in non-bonding interactions with several prosthetic groups present in the enzyme cavity. Interesting work by Sheng and co-workers, involving protein docking studies, demonstrated that organic modification with different functional groups at the C3 atom of fluconazole resulted in an increased activity in relation to the parent drug. This was supported by $\mathrm{MIC}_{80}$ values. Therefore, in the present case, we aimed at derivatizing the fluconazole core on the triazole not involved in the binding pocket interaction, replacing it with a ferrocenyl moiety. Ferrocenyl derivatization is proposed to play an important role for the overall biodistribution and uptake (e.g. increasing the lipophilicity of the parent drug) as well as in potentially allowing for an additional redox-induced mode of action as observed for ferrocifen and ferroquine. ${ }^{32,57,58,61}$ Finally, different alkyl substituents were inserted at the bridging nitrogen in order to garner Structure-Activity Relationship (SAR) information. The target ferrocenyl compounds of this study, namely 1a-4a can be seen in Fig. 1. Herein, we name them fluconazenes.

The synthesis of the new fluconazenes 1-4 can be visualized in Scheme 1. In the envisioned retrosynthesis (Scheme 1A), the target compounds would be obtained from the addition of a ferrocenylamine (5) on the epoxide 6, easily accessible from 1,2,4-triazolo-ketone 7, which is commercially available, by a Corey-Chaykovski epoxidation. The ferrocenylmethanamines (5a-d) were prepared from ferrocene carboxaldehyde (8) in two steps, following adapted procedures by Tice et al. and Baramee et al. ${ }^{62,63}$ Reaction of the aldehyde (8) with hydroxylamine hydrochloride under basic conditions yielded the oxime (9a). The imines (9b-d) were obtained by stirring 8 in the presence of a solution of the corresponding alkyl amines. The intermediates $(\mathbf{9 a}-\mathbf{d})$ were reduced to amines $(\mathbf{5 a}-\mathbf{d})$ with $\mathrm{LiAlH}_{4}$ and $\mathrm{NaBH}_{4}$, respectively. Spectroscopic data of 5a-d matched those reported in the literature (Scheme 1B). ${ }^{62-64}$ Epoxide 6 was obtained in $76 \%$ yield by treating ketone 5 with a solution of trimethylsulfoxonium iodide ylide (Scheme 1C). ${ }^{60,65}$ The subsequent epoxide ring opening of $\mathbf{6}$ with the primary and secondary ferrocenemethanamines (5a-d) yielded compounds 1-4. ${ }^{64,65}$ The derivative carrying the methylamine linker (2) was obtained in noticeably higher yield (46\%) than the other derivatives $(1,30 \% ; 3$, $31 \% ; 4,20 \%)$. This can be attributed to the donating inductive effect of the alkyl groups on the $\mathrm{N}$ atom, which increases the nucleophilic strength of $\mathbf{9 b - d}$. In contrast, the growing steric hindrance of the ethyl and isopropyl group can explain the decreasing yield within the series of tertiary amines $(2>3>4)$.

While fluconazole is achiral, with two identical 1,2,4-triazolylmethyl side chains attached to the $\mathrm{C} 2$ carbon, our derivatives 1-4 are chiral. However, according to molecular docking experiments of chiral fluconazole derivatives by Sheng et al., both $R$ and $S$ isomers, can interact with the active site of the $C$. albicans CYP51 through a similar binding mode, ${ }^{66}$ thus avoiding the need for a chiral separation, or an asymmetric synthesis of a specific enantiomer. ${ }^{67}$ The derivatives 1-4 were converted to the corresponding hydrochloride salts by treatment with $\mathrm{HCl}$ in acetone, following an adapted procedure of Bader et al. (Scheme 1D). ${ }^{68}$ Overall, the new complexes $\mathbf{1 a}-\mathbf{4 a}$ were all characterized via ${ }^{1} \mathrm{H}$,
${ }^{13} \mathrm{C},{ }^{19} \mathrm{~F}$ NMR, MS, IR and their purity was confirmed via elemental analysis and UPLC-MS (see Fig. S2-S29 in ESI $\dagger$ ).

\section{Stability}

As potential drug candidates, the hydrochloride salts 1a-4a need to be stable in DMSO, which is the administering medium for in vitro and in vivo studies, as well as in the biological medium, namely water. It was previously demonstrated that this can be problematic for metal-based drugs. ${ }^{69-71}$ The stability of 1a-4a was assayed by dissolving the salts in deuterated solvents, namely neat DMSO- $\mathrm{d}_{6}$ and neat $\mathrm{D}_{2} \mathrm{O}$, and by monitoring the samples using ${ }^{1} \mathrm{H}$ NMR spectroscopy over a period of 48 hours (see $\mathrm{ESI} \dagger$ for details). It is worth mentioning that $\mathbf{1 a}$ and $\mathbf{4 a}$ are only sparingly soluble in $\mathrm{D}_{2} \mathrm{O}$. Generally, all four hydrochloride salts were stable for up to 24 hours and only little decomposition was observed for $\mathbf{2 a}$ and $\mathbf{4 a}$ in water after $48 \mathrm{~h}$.

\section{In vitro biological screening}

To assess the antifungal activity of the new drug candidates, we employed a well-plate based assay recently developed in our group. ${ }^{39}$ The first antimycotic investigation was performed on the solid and largely used model system $S$. cerevisiae. Therefore, $S$. cerevisiae was cultured and diluted to reach the beginning of the growing phase just before treatment. YPD agar terrain at increasing concentrations of the target complexes were poured on a 6 or 12-wells plate and the $S$. cerevisiae culture were then spotted on them and incubated for $24 \mathrm{~h}$ at $30{ }^{\circ} \mathrm{C}$. Interestingly, all compounds displayed an improved antifungal activity compared to the parent drug (e.g. see Fig. 2). Small alkyl group-substituted tertiary amines (complexes 2a and 3a) as linkers, showed an improved profile, between 9-17-fold, compared with secondary amines (see Table 1).

To further evaluate the potential of the compounds, we investigated their host toxicity, in vitro mycotoxicity and cellular uptake. An effective inhibitor would need a good therapeutic window, with low host toxicity versus effective nM fungal toxicity The first investigation on the new antimycotic drug candidates involved their possible influence on host cells and their efficiency towards a common fungal model. We investigated the antiproliferative effect of 1a-4a in vitro on human retinal pigment epithelial cell (RPE-1-hTert) and human fibroblast (MRC-5). As expected, fluconazole displayed only moderate effects on cell viability. All complexes displayed $\mathrm{IC}_{50}$ values in the mid-high micromolar range, following the order of potency: isopropyl (4a) > ethyl (3a) > methyl (2a) > H (1a) (see Table 1). This suggests that a decrease in the size of the $N$-substituent (i.e., a decrease in lipophilicity) correlates with a decrease in host toxicity. Overall, while the organometallic compounds are slightly more toxic than fluconazole, the toxicity remains relatively moderate, especially taking into account that the toxicity towards yeast is much higher (see below).

In order to investigate the pharmaco-dynamic profile of the target antimycotic drug candidates, a series of uptake studies in $S$. cerevisiae were performed. The uptake measurements were performed via ICP-MS by detecting the free iron content in the culture medium and normalizing it against the colony density 
A.

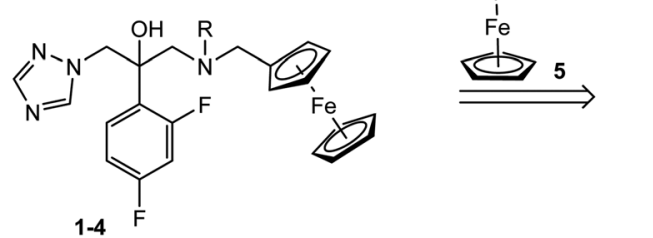

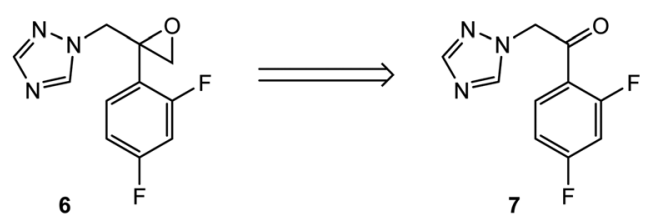

B.

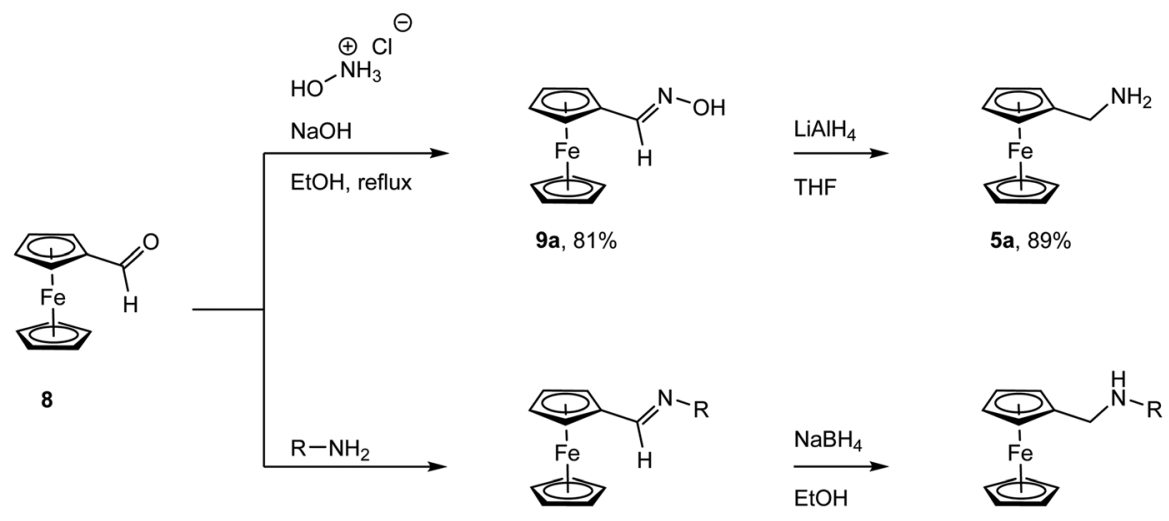

9b: $\mathrm{R}=\mathrm{Me}, 74 \%$

9c: $R=E t, 90 \%$

9d: $\mathrm{R}=i-\mathrm{Pr}, 92 \%$ 5b: $\mathrm{R}=\mathrm{Me}, 76 \%$

5c: $\mathrm{R}=\mathrm{Et}, 67 \%$

5d: $\mathbf{R}=i-\operatorname{Pr}, 89 \%$

c.<smiles>C[Sn](C)(C)O[Na]</smiles>

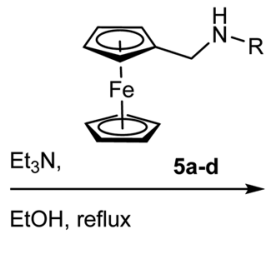

D.<smiles>[R]N(Cc1cccc(PC2=CC=C2)c1)CC(O)(Cn1cncn1)c1ccc(F)cc1F</smiles><smiles>[R]N(Cc1ccc(Pc2ccco2)cc1)CC(O)(Cn1cncn1)c1ccc(F)cc1F</smiles>

1a: $\mathrm{R}=\mathrm{H}, 82 \%$

2a: $R=M e, 82 \%$

3a: $R=E t, 93 \%$

4a: $\mathrm{R}=i-\operatorname{Pr}, 77 \%$

Scheme 1 (A) Retrosynthetic analysis; (B) preparation of the primary (7a) and secondary (7b-d) ferrocenylmethanamines via the oxime (9a) and imine (9b-d) intermediates. Overall yields, comprising both steps: 7a, 71\%; 7b, 56\%; 7c, 60\%; 7d, 81\%; (C) synthesis of the organometallic fluconazole derivatives (1-4), which feature an amine-linked ferrocene moiety; (D) synthesis of the hydrochloride salts of 1-4 (1a-4a).

at different incubation times $(1 \mathrm{~h}, 6 \mathrm{~h}$ and $18 \mathrm{~h})$. The results were then compared with the survival rate measured via OD (see Fig. 3). The uptake of the target complexes followed the order $\mathbf{4 a} \ll \mathbf{1 a}<$ $3 \mathbf{a}<2 \mathbf{a}$ and fits very well with their time-dependent mycotoxicity. This suggests that complexes with sterically-hindered groups like an isopropyl or the less lipophilic complex 1a showed a lower efficacy than ones with short chain alkyl groups (2a, 3a).
In order to assess the possible increase in reactive oxygen species (ROS) level induced by 1a-4a, possibly due to the presence of the ferrocenyl moiety, we performed a series of in vitro experiment to measure the ROS level upon treatment on S. cerevisiae. Briefly, S. cerevisiae was cultured in YPD medium overnight before treatment. After that, the fungal concentration was measured and normalized and the cultures were treated 

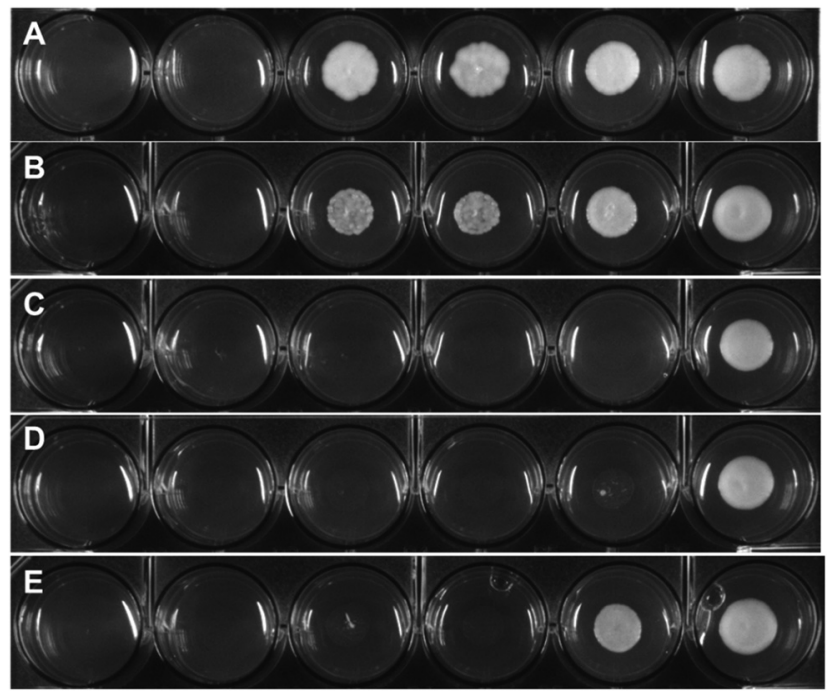

Fig. 2 Depicted example of a colony formation inhibition experiment; (A) S. cerevisiae treated with fluconazole; (B) S. cerevisiae treated with 1a; (C) S. cerevisiae treated with $2 \mathbf{a}$; (D) $S$. cerevisiae treated with $3 \mathbf{a}$; (E) $S$. cerevisiae treated with $\mathbf{4 a}$. S. cerevisiae has been treated with increasing concentrations of the target drug candidates ranging from 2.5 up $200 \mu \mathrm{M}$ (higher concentration not depicted) and incubated at $30{ }^{\circ} \mathrm{C}$ for $24 \mathrm{~h}$. The experiments with the drug candidates and with negative control of untreated colony have been performed in triplicate.

Table 1 Host toxicity of the new fluconacenes against RPE and MRC-5 human cell lines (expressed as $I C_{50}$ ) and inhibition of colony formation in $S$. cerevisiae treated with increasing concentrations of the $1 \mathrm{a}-4 \mathrm{a}$ for $24 \mathrm{~h}$ at $30{ }^{\circ} \mathrm{C}$; fluconazole was used as parent drug for comparative purposes; values are expressed in $\mu \mathrm{M}$. The activity of compounds $1 \mathrm{a}-\mathbf{4 a}$ on $\mathrm{S}$. cerevisiae was evaluated via a newly established colony formation assay $^{39}$

\begin{tabular}{llll}
\hline Compound & MRC5 & RPE & S. cerevisiae \\
\hline Fluconazole & $>100$ & $>100$ & $51.0 \pm 3.7$ \\
1a & $94.6 \pm 2.4$ & $>100$ & $13.2 \pm 3.0$ \\
2a & $32.2 \pm 0.1$ & $74.7 \pm 5.2$ & $3.02 \pm 2.18$ \\
3a & $70.4 \pm 17.9$ & $51.2 \pm 0.1$ & $5.86 \pm 0.41$ \\
4a & $59.4 \pm 2.8$ & $48.4 \pm 2.9$ & $9.62 \pm 0.56$ \\
\hline
\end{tabular}

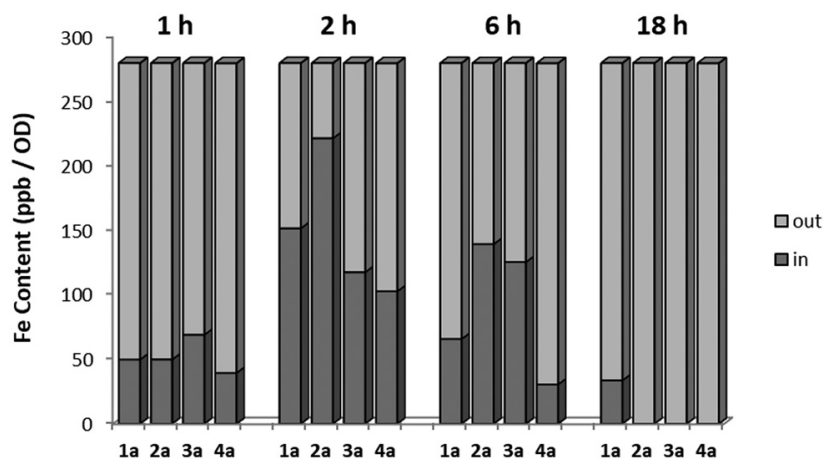

Fig. 3 Uptake studies compared to the colony survival rate measured in OD. The experiment has been performed on the medium of incubation and corrected with $\mathrm{OD}$ in order to take into account of the possible biological material loss due to toxicity of the compounds and which would lead to decrease in uptake. Negative controls of the untreated and of the medium alone have been also performed. with YPD containing $5 \mu \mathrm{M}$ of the different drug candidates at different time frames ( $1 \mathrm{~h}, 6 \mathrm{~h}$ and $18 \mathrm{~h})$. After incubation, the colonies were washed, fresh medium containing H2DCF was added and the fluorescence of the hydrolyzed DCF was measured (see Fig. S1, ESI $\dagger$ ). While the parent drug fluconazole displayed weak action at early stages, which increased with incubation time, the new drug candidates showed a higher ROS levels at an early stage. However, although the complexes proved to induce an increase of ROS levels, the relative ROS induction still remains moderate and it is probable that ROS production is not responsible for fungi death.

\section{Morphology studies}

The activity of $\mathbf{2 a}$ at the biochemical level is also mirrored by morphologic features at the ultra-structural level. The morphologic effect on $S$. cerevisiae upon treatment with the target complex could be conveniently monitored by transmission electron microscopy (TEM). As can be seen in Fig. 4, the resulting images (selection of a large pool) suggested that $2 \mathrm{a}$ induces a loss in wall integrity as well as intracellular features ascribable to late apoptosis or necrosis.

\section{In vitro antifungal screening on clinical isolates}

Motivated by the promising results obtained for the in vitro antifungal activities against the non-pathogenic model system $S$. cerevisiae, we performed further screening on clinical isolates. We again chose complex 2a since it displayed the best mycotoxicity and, on the other side, showed low cytotoxicity against human cells. We tested this antifungal drug candidate towards $C$. albicans and non albicans strains, including fluconazole-resistant $\left(\mathrm{MIC}_{50}>\right.$ $100 \mu \mathrm{M})$ and other mycotoxin-producing and pathogenic fungi, such as Penicillum paneum, Aspergillus glaucus and Trichosporon asahi (see Table 2). Of utmost interest, the new antifungal drug candidate displayed a very strong activity towards almost all investigated strains (only in the case of A. glaucus, C. parapsilosis, and $C$. albicans MFB005 and SC5314 was the $\mathrm{MIC}_{50}$ not in the nanomolar range). Moreover, the antimycotic potency of the complex did overcome the values of the parent drug up to a factor of 400-fold (e.g. P. paneum) and also showed activity towards azole resistant strains.

Fluconazole is a fungistatic drug and growth curve analysis performed on the C. albicans reference strain SC5314, a frequently used wild-type control, indicate a fungistatic effect of compound $2 \mathbf{a}$ at $\mathrm{MIC}_{50}$ concentration for this specific strain (1.98 $\mu \mathrm{M}$, Fig. S2, ESI $\dagger$ ).

\section{AOX enzyme inhibition}

In order to further define the inhibitory capabilities of the compounds, they were also tested in the mitochondrial complex II and III pathway (succinate quinol reductase pathway (SQR)), two common targets for fungicidal treatment. ${ }^{72,73}$ As can be seen in Fig. 5, compound 1a demonstrated no inhibitory activity towards either complex, confirming that the antifungal properties of these compounds are not due to off-target effects within mitochondrial respiration. However, while the other analogues demonstrate slightly improved inhibitory properties against the pathway when compared to 1a, it is unlikely to be the source of fungicidal activity. 

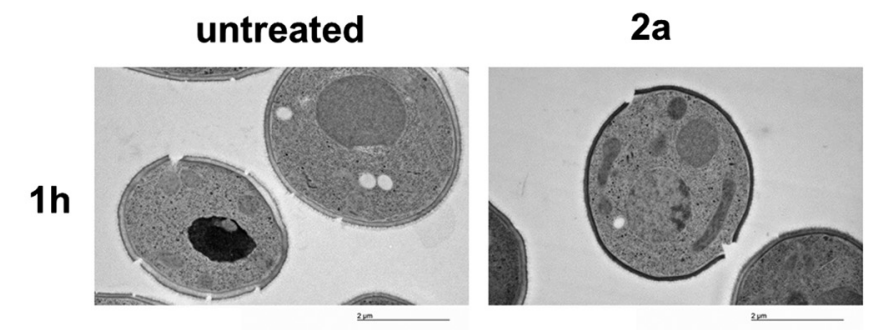

fluconazole
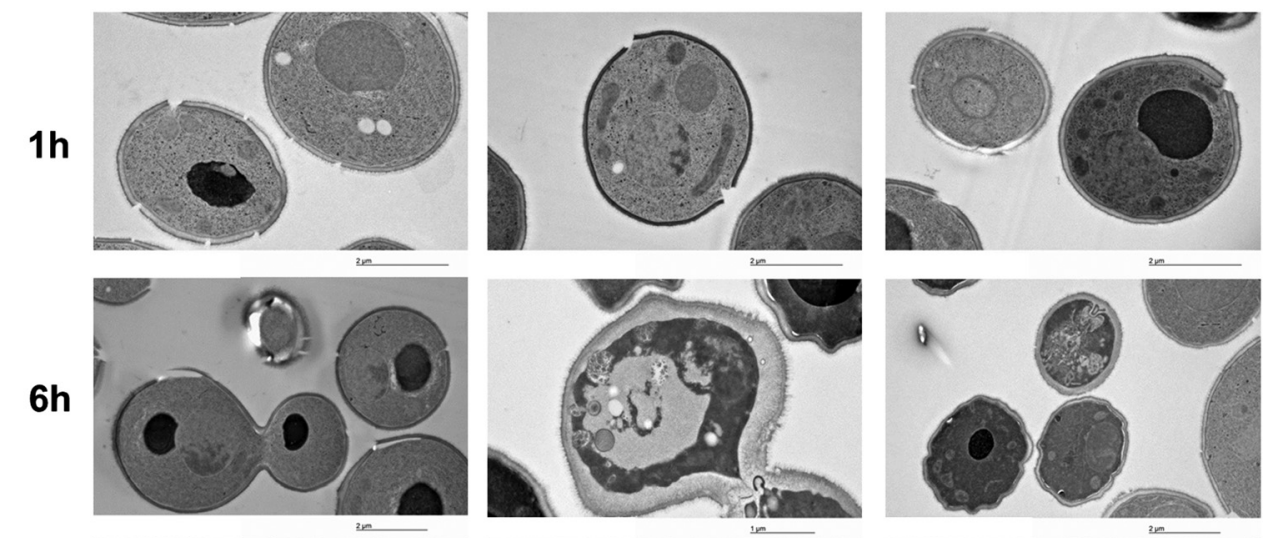

$18 \mathrm{~h}$
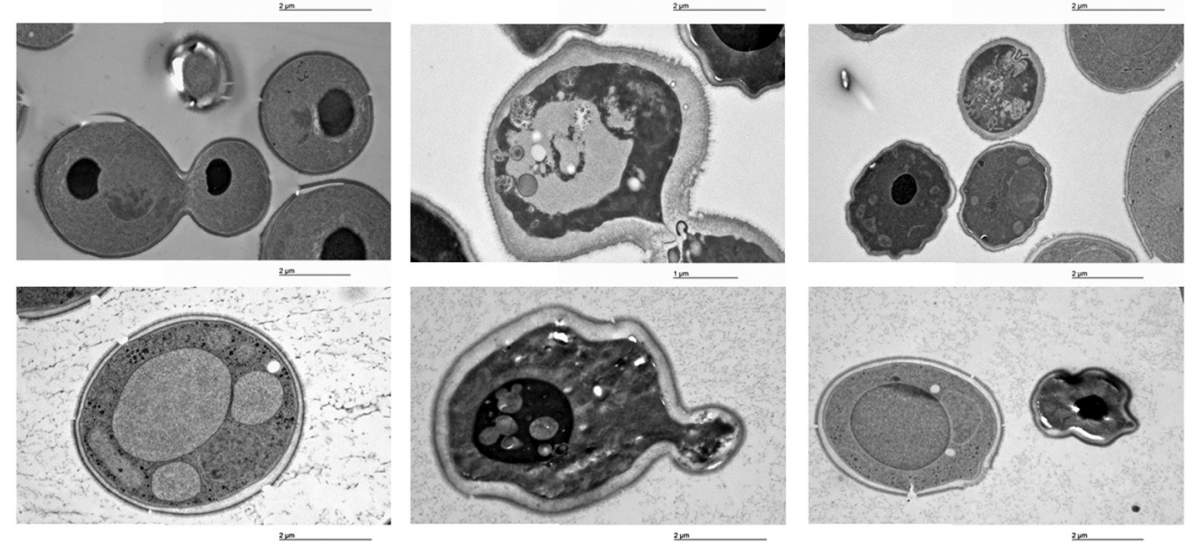

Fig. 4 Ultra-structural studies of untreated S. cerevisiae, treated with $\mathbf{2 a}$ or with fluconazole tested at $5 \mu \mathrm{M}$ and $30{ }^{\circ} \mathrm{C}$ incubation temperature. The pictures showed starting with a $6 \mathrm{~h}$ incubation formation of vesicles, ascribable as apoptotic bodies and loss of membrane integrity. After longer incubation times a shrinking effect as well as nuclear blebbing could be also noted.

Table $2 \quad$ MIC $_{50}$ values on a pool of fungal pathogens treated with $2 a$

\begin{tabular}{lll}
\hline Strains & $\mathrm{MIC}_{50} 2 \mathrm{a}(\mu \mathrm{M})$ & $\mathrm{MIC}_{50}$ fluconazole $(\mu \mathrm{M})$ \\
\hline C. albicans SC5314 & $>1$ & 0.82 \\
C. albicans MFB005 FS3 & $>1$ & 0.41 \\
C. albicans YMS 102-2 & 1.00 & $>100$ \\
C. albicans YMS 102-6 & 0.06 & 0.82 \\
C. glabrata MFB005 FS4 & 0.06 & 0.41 \\
C. glabrata RTT 199_3 & 1.00 & $>100$ \\
C. parapsilosis MFB005 FS5 & 0.50 & 0.41 \\
C. parapsilosis MFB070 N1 & $>1$ & $>100$ \\
C. tropicalis RTT35-1 & 0.69 & $>100$ \\
C. tropicalis RTT35-3 & 0.50 & $>100$ \\
P. paneum MFB042 N1 & 0.25 & $>100$ \\
A. glaucus MFB027 N1 & $>1$ & 0.82 \\
T. asahii MFB034 N1 & 0.13 & 1.63 \\
\hline
\end{tabular}

The compounds were also tested against alternative oxidases (AOX) from both $C$. albicans and $C$. auris. The proteins were expressed in a recombinant $E$. coli system to allow for more accurate data acquisition due to their low expression levels proving problematic from a detection standpoint. Again, compound 1a demonstrated minimal inhibitory activity towards the AOX's, however compounds 3a and 4a showed moderate activity against both AOX's, comparable to values typically seen with non-competitive AOX inhibitors such as salicylhydroxamic acid (SHAM). ${ }^{74}$ The parent compound, fluconazole, displayed no inhibition towards either pathway up to a concentration of $100 \mu \mathrm{M}$.

\section{Cytochrome P450 enzyme inhibition}

Based on the very promising data obtained with complex $\mathbf{2 a}$, further biological experiments were performed on this complex

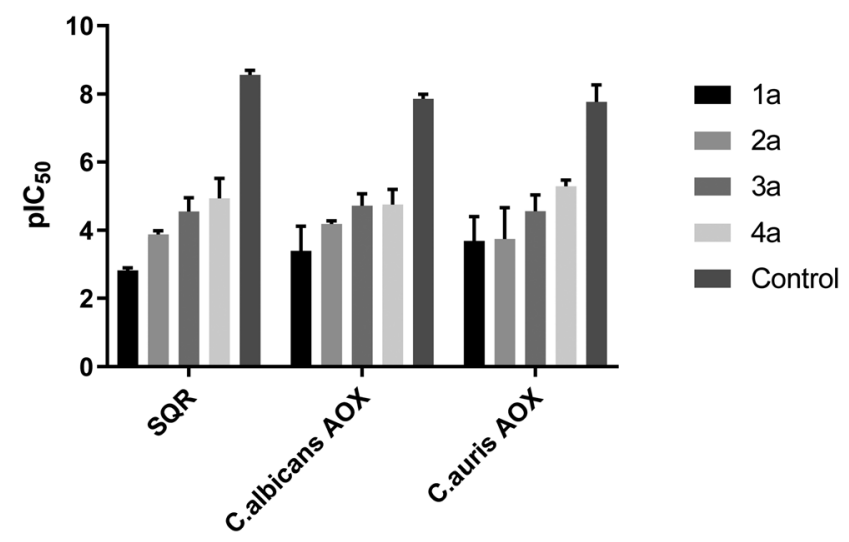

Fig. 5 plC 50 values $\left(-\log \mid C_{50}\right)$ for all compounds against the mitochondrial complex II and III pathway (SQR) from rat liver mitochondria, and rAOX's from C. albicans and C. auris. Control denotes control compounds for each specific pathway, with antimycin A for SQR and ascofuranone for the AOX pathways. Data was collected in a 96 -well plate format, following cytochrome $c$ reduction $\left(\triangle A^{550}\right)$ for the SQR pathway, and NADH oxidation $\left(\Delta A^{340}\right)$ for the AOX pathway. All data are a mean average of 3 biological replicates \pm SEM.

to understand further its mechanism of action. Enzyme inhibition studies were performed on different cytochrome P450 enzymes by the service provider Cyprotex $\mathrm{GmbH}$. The new fluconacene 2a displayed an extremely strong inhibition profile throughout all the series of enzymes, with a larger efficacy in comparison with the parent drug fluconazole, between 4 and 150 fold (see Table 3) suggesting that, if this series of molecule are optimised towards clinical use, like fluconazole ${ }^{75}$ potential drug-drug interactions will need to be taken into account. 
Table 3 Enzyme Inhibition studies of different cytochrome P450 enzyme by the compound $2 \mathbf{a}$ and its parent drug (IC 50 in $\mu \mathrm{M})$. Standard deviation shown in upper script

\begin{tabular}{llllll}
\hline Compound & CYP2C9 & CYP2C8 & CYP2C19 & CYP3A4 & CYP1A \\
\hline Fluconazole & $13.9 \pm 1.63$ & $97.2 \pm 27.9$ & $3.02 \pm 0.34$ & $25.8 \pm 2.45$ & $>100$ \\
2a & $3.33 \pm 0.71$ & $0.64 \pm 0.09$ & $<0.4$ & $0.4 \pm 0.06$ & $10.4 \pm 0.41$
\end{tabular}

\section{Chemogenomic studies}

In order to investigate the mechanism of action of the new organometallic complex 2a, chemogenomic assays were performed. The strength of the genome-wide chemogenomic screen is reflected in the clear identification of ERG11, encoding lanosterol 14-alphademethylase, as the main target of the drug (see Fig. 6 and Table S1, ESI $\dagger$ ). ERG11 is a key enzyme of the ergosterol biosynthesis pathway and the primary target of azole antifungals to which the tested compound 2a belongs.

Azoles are known to induce $E R G 11$ gene expression, ${ }^{76}$ which also holds true for compound $2 \mathrm{a}$ yet to a lower extent than fluconazole as revealed by a screen in both susceptible C. albicans strains (Fig. S3A, ESI $\dagger$ ) and in the highly fluconazole resistant strain YMS102-2 (Fig. S3B, ESI $\dagger$ ). The latter strain showed a high expression of ERG11 when grown in the absence of drug and $E R G 11$ gene expression was significantly $(\mathrm{FC}=1.69$; $P=0.000086)$ increased upon fluconazole exposure, while decreased (FC $=-1.81 ; P=0.000193$ ) when grown in the presence of compound 2a (Fig. S3B, ESI $\dagger$ ). Hence, the improved antifungal activity of compound 2a for the strain YMS102-2 might be partially explained by the significant reduction of ERG11 gene expression, because overexpression of ERG11 is known as an azole resistance mechanism in Candida. ${ }^{16,77}$
Hence, future work should be directed to study if compound 2a is able to reduce ERG11 gene expression also in other ERG11 overexpressing Candida isolates in a similar way.

Our chemogenomic assay identified five other heterozygous deletion strains that in previous chemogenomic screens have been shown to be extremely sensitive to azole antifungals; these are SET6, PDR5, PDR16, MED4 and CDC39. The latter two are essential nuclear genes involved in the regulation of transcription. CDC39 acts as a component of the CCR4-NOT core complex ${ }^{78}$ and MED4 encodes a subunit of the RNA polymerase II mediator complex. In Saccharomyces cerevisiae and C. glabrata the mediator complex is a coactivator of the multiple drug resistance regulator PDR1 that controls the activation of PDR5 and PDR16. ${ }^{79-81}$ Similarly, also in C. albicans the mediator activator complex is recruited to the CDR1 promotor, ${ }^{80}$ which is the C. albicans ortholog of PDR5. PDR5 is a multidrug $\mathrm{ABC}$ efflux pump that confers resistance to several chemicals including azoles and it mediates transmembrane transport of steorids. ${ }^{82}$ PDR16 (ortholog of the C. albicans $P D R 17$ ) is a phosphatidylinositol transfer protein involved in sterol biosynthetic process and in resistance to azole drugs. ${ }^{83}$ Notably, the phosphatidylinositol signal transduction pathway that controls amongst others cell membrane and cell wall remodelling as well as downstream targets such as DNA repair, was recently suggested to

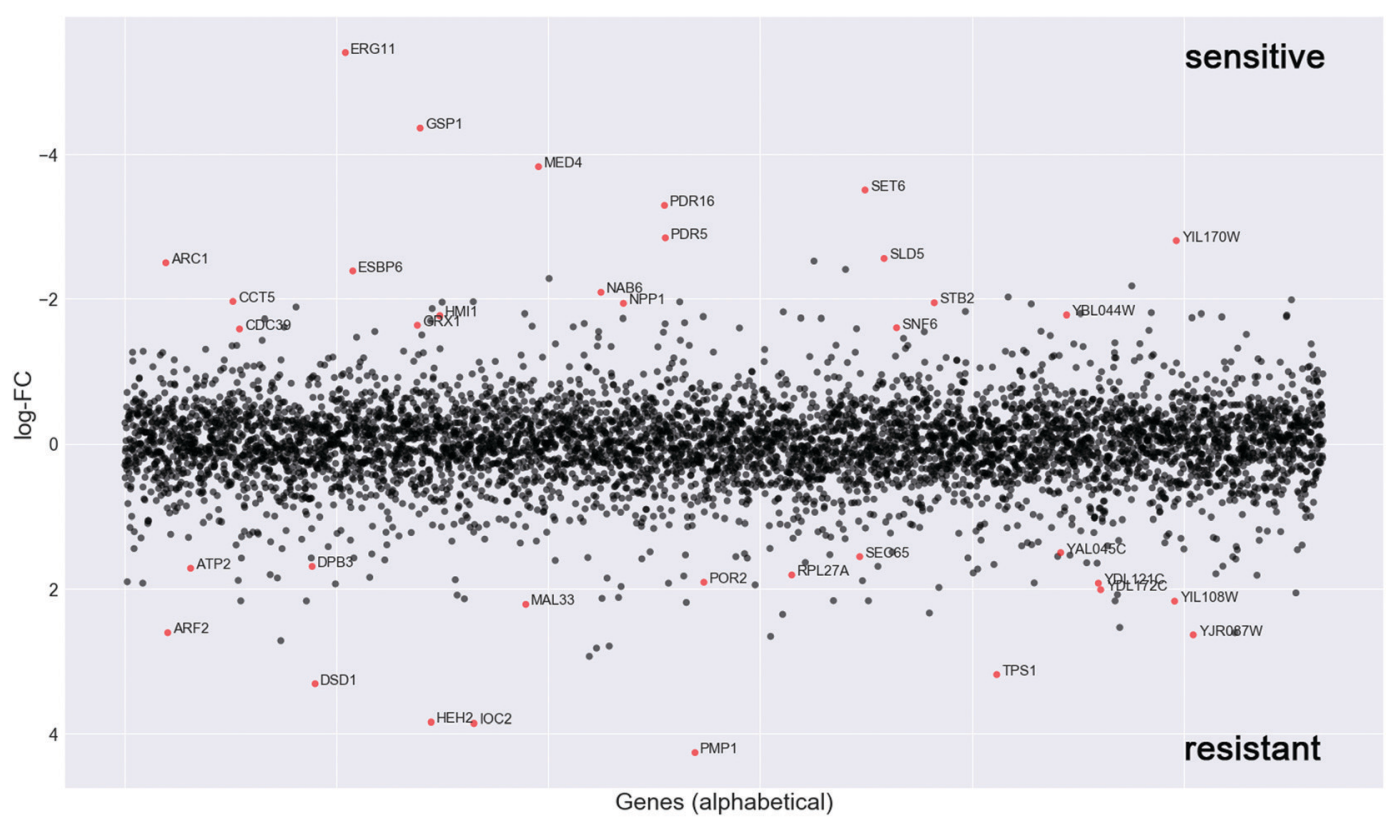

Fig. 6 Chemogenomic screen. To identify potential drug targets the pool of tagged 5936 S. cerevisiae heterozygous deletion mutants was grown competitively for 20 generations in the presence and absence of compound 2a. Strain fitness was determined via high-throughput barcode sequencing and normalization to the untreated control. $\log 2$ ratio (control intensity/treatment intensity) was calculated and plotted as a function of gene. The genome-wide readout of heterozygous mutants highly sensitive to compound $\mathbf{2 a}$ included the known target of azoles, ERG11 and novel targets such as the RAN GTPase GSP1 (red dots: log FC > 1.5 and $P$ value $>0.01$ ). 
play an important role in phenotypic antifungal drug resistance related to protein synthesis. ${ }^{18}$ Indeed many of the identified deletion strains that are highly sensitive to compound 2a were heterozygous for genes involved in protein synthesis (e.g. ARC1, RPS5, MED4, CDC39, SNF6, CCT5, GSP1) (Fig. 4 and Table S1, ESI $\dagger$ ).

Furthermore, compound 2a targets nuclear genes that are not only essential for RNA transcription, processing and transport (GSP1, MED4, CDC39) but also essential for DNA replication ( $S L D 5$ ), and these together with gene targets functioning in the correct folding of actin and tubulin (CCT5) (Fig. 6) point to a possible interaction of compound $2 \mathrm{a}$ with cell division.

Taken together, the genome-wide profiling of the in vivo cellular response to compound $\mathbf{2 a}$ identified candidate protein targets (Fig. 6 and Table S1, ESI $\dagger$ ), which hint to a mechanism
A
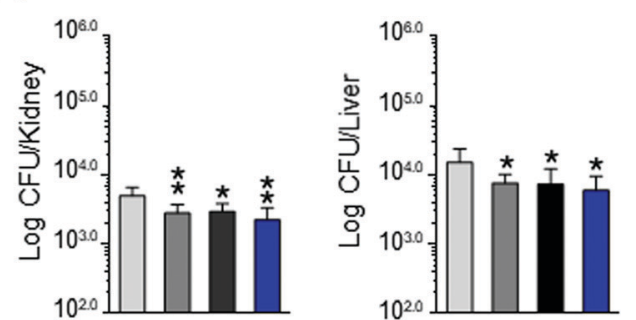

C
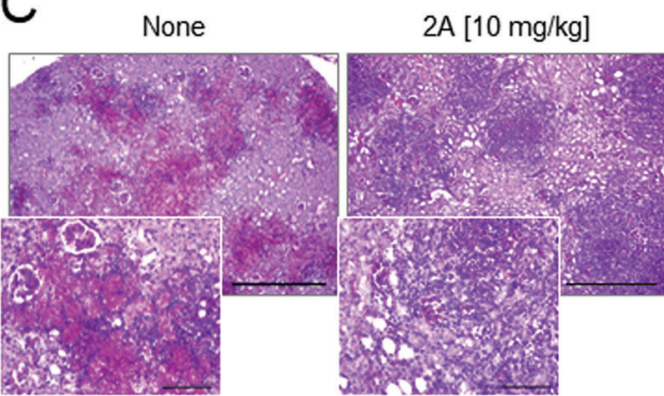

B

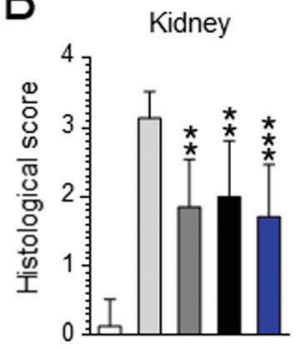

$2 \mathrm{~A}[1 \mathrm{mg} / \mathrm{kg}]$
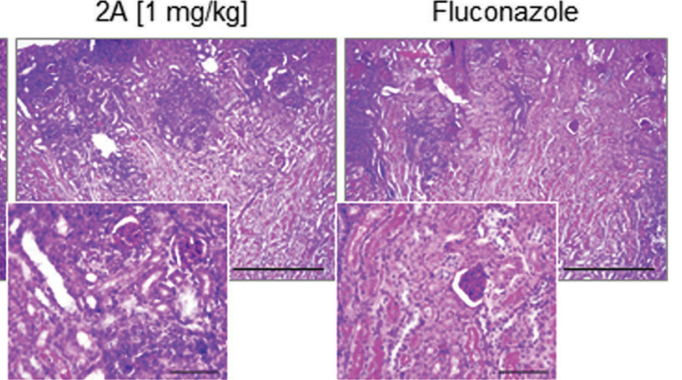

D

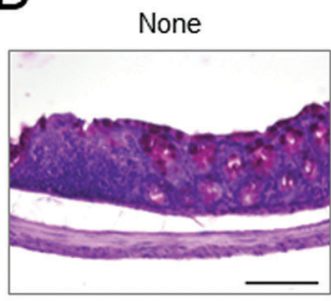

$2 \mathrm{~A}[10 \mathrm{mg} / \mathrm{kg}]$
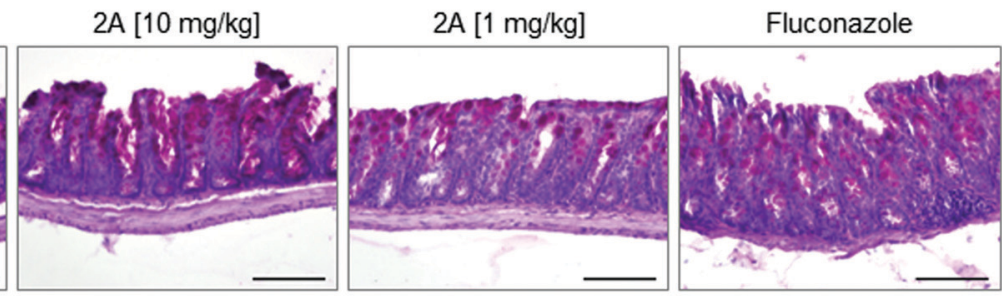

$\mathrm{E}$

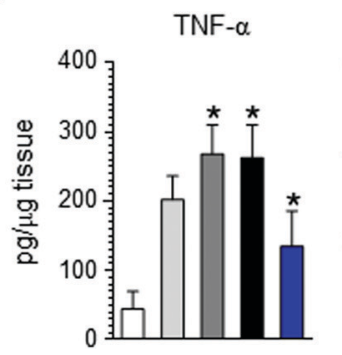

IL-1 $\beta$
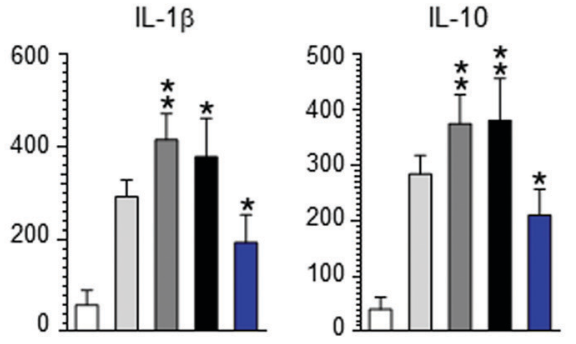

Colon

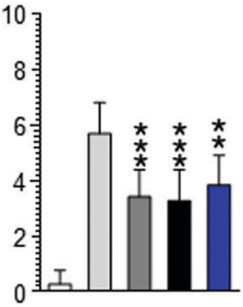

Fluconazole

Fig. 7 Effect of the compound 2a on C. albicans (SC-5314) systemic infection. C57BL/6 mice were infected via systemic route with $1 \times 10^{6}$ C. albicans yeasts. Fluconazole and compound $\mathbf{2 a}$ were administered intragastrically daily, beginning the day of the infection until mice sacrifice $(4$ days after the infection). Control mice received the diluent alone. (A) Fungal growth. (B) Histological scores and (C and D) analysis (10× magnification and $40 \times$ in the inset) of the kidneys and colon of infected and treated mice. (E) Levels of cytokines in kidney homogenates. For histological scores of kidneys, score $0=$ no inflammation, score $1 \leq 3$ foci of inflammation, score $2=4$ to 6 foci of inflammation, score $3 \geq 6$ foci of inflammation, but less than $25 \%$ of kidney affected, score $4 \geq 25 \%$ of kidney affected. For histological scores of colon, the total histological score was obtained by summing the four histological components scores: 'inflammation extent', 'damage in crypt architecture', 'hyperemia/edema', 'grade of accumulation with inflammatory cells'. Data are expressed as mean $\pm \mathrm{SD}$. ${ }^{*} P<0.05,{ }^{*} P<0.01,{ }^{*} * P<0.005$, treated $v$ s. untreated C57BL/6 mice $(n=7$ mice per group, representative of three experiments). Naïve, uninfected mice. None, untreated mice. 
of drug action that goes beyond the main targets of azole antifungals, which might explain its elevated activity towards azole resistant strains (Table 1).

\section{In vivo studies}

Motivated by these promising results, we decided to perform in vivo experiments in a mice model of Candida infection. As positive control, fluconazole was used. It significantly improved pathology and cured mice from the infection, as judged by the decreased fungal growth in the kidney and dissemination to the liver (see Fig. 7A), reduction of the inflammatory pathology in the kidney and colon (see Fig. 7B-D) and downregulation of pro-inflammatory cytokine (IL-1 $\beta$ and TNF- $\alpha$ ) levels (Fig. 7D). Similar results were obtained with compound 2 a that proved to be effective in reducing fungal growth and dissemination (see Fig. 7A) as well as tissue inflammatory pathology (see Fig. 6B for histological scores) at a dose as low as $1 \mathrm{mg} \mathrm{kg}^{-1}$ (Fig. 7C and D). Of great interest, at variance with fluconazole, compound 2a also promoted a vigorous pro-inflammatory cytokine response promptly controlled by the associated high levels IL-10 (Fig. 7E). These findings indicate that compound $\mathbf{2 a}$ is active in vivo and that its activity may encompasse an action on innate immune response, consistent with the well known synergistic activity of azoles, including fluconazole, with antifungal effector activities, such as phagocytosis, radicals production and Toll-like receptors activation. ${ }^{84}$

\section{Conclusion}

Four novel ferrocene-based derivatives (1a-4a) of the frontline antifungal drug fluconazole have been synthesized and characterized. All four organometallic derivatives were tested for antifungal activity against the model system $S$. cerevisiae and showed an impressively improved activity compared to fluconazole. Importantly, this activity was confirmed on a panel of clinical isolates with the best candidate of this study (complex 2a). An improvement of the minimum inhibitory concentration of up to 400 times compared to fluconazole was observed and activity against azole-resistant strains was clearly demonstrated for $\mathbf{2 a}$. During in vivo experiments in mice model of Candida infections, compound 2a was found to diminish the growth of the fungus and to greatly improve the inflammatory pathology in the kidney and colon. Overall, this study further demonstrates the potential of organometallic compounds in medicine. By merely inserting a ferrocenyl moiety in a well-known drug, namely fluconazole, resistance can be overcome, although the main target of the novel antimycotic agent is the same as that of the organic drug (lanosterol $14 \alpha$-demethylase), as demonstrated by chemogenomic profiling. The ferrocenyl insertion allows for an additional mode of action.

\section{Ethical statement}

Mouse experiments were performed according to Italian Approved Animal Welfare Authorization 360/2015-PR and Legislative Decree 26/2014 regarding the animal license obtained by the Italian
Ministry of Health lasting for 5 years (2015-2020). Animals were treated as the guidelines of EAMC.

\section{Author contributions}

All authors were involved with the design and interpretation of experiments and with the writing of the manuscript. The synthesis and characterization of the compounds were performed by R. R., L. M., S. J., J. N., Y. L., and J. H. TEM studies were carried out by A. K. Biological experiments were performed by R. R., T. W., N. T., A. L. M., and L. Y. Chemogenomic studies were carried out by T. W. and M. M. Animal testing was carried out by R. G., M. P. and L. R. All authors have given approval to the final version of the manuscript.

\section{Conflicts of interest}

The authors declare no competing financial interests.

\section{Acknowledgements}

This work was funded by the Swiss National Science Foundation (Grant Sinergia CRSII5_173718, Professorships No. PP00P2_133568 and PP00P2_157545 to G. G.), the University of Zurich (G. G.), the Stiftung für Wissenschaftliche Forschung of the University of Zurich (G. G.), the Novartis Jubilee Foundation (G. G. and R. R.), the Forschungskredit of the University of Zurich (R. R.) and the UBS Promedica Stiftung (G. G. and R. R.). This work has received support under the program "Investissements d'Avenir" launched by the French Government and implemented by the ANR with the reference ANR-10-IDEX0001-02 PSL (G. G.). This research was supported by the Autonomous Province of Trento (Accordo di Programma P1611051I (M. M.)). Work in A. L. M.'s laboratory is supported by BBSRC (BB/L022915/1 and BB/NO10051/1). The authors thank Monica Borghi for her technical assistance for the mice experiments. We would also like to thank the University of Sussex (HEIF COVID-19 emergency funds) and the Ewart Bequest Fund for this work (J. S.).

\section{References}

1 T. Ohyama, S. Miyakoshi and F. Isono, Antimicrob. Agents Chemother., 2004, 48, 319-322.

2 G. Maschmeyer, Int. J. Antimicrob. Agents, 2006, 27S, S3-S6.

3 A. Mikolajewska, S. Schwartz and M. Ruhnke, Mycoses, 2012, 55, 2-16.

4 R. Pelletier, J. Peter, C. Antin, C. Gonzalez, L. Wood and T. J. Walsh, J. Clin. Microbiol., 2000, 38, 1563-1568.

5 K. Donhuijsen, P. Petersen and W. K. Schmid, Dtsch. Arztebl. Int., 2008, 105, 501-506.

6 P. N. Lipke and R. Ovalle, J. Bacteriol., 1998, 180, 3735-3740.

7 S. M. Bowman and S. J. Free, BioEssays, 2006, 28, 799-808.

8 S. Perea, M. J. Ramos, M. Garau, A. Gonzalez, A. R. Noriega and A. del Palacio, J. Clin. Microbiol., 2000, 38, 3226-3230. 
9 M. P. English and J. Turvey, Br. Med. J., 1968, 4, 228-230.

10 B. Havlickova, V. A. Czaika and M. Friedrich, Mycoses, 2008, 51(Suppl. 4), 2-15.

11 R. P. Hobson, J. Hosp. Infect., 2003, 55, 159-168.

12 D. A. Enoch, H. A. Ludlam and N. M. Brown, J. Med. Microbiol., 2006, 55, 809-818.

13 J. Menzin, J. L. Meyers, M. M. Friedman, J. R. Korn, J. R. Perfect, A. A. Langston, R. P. Danna and G. Papadopoulos, Am. J. Infect. Control, 2011, 39, 15-20.

14 R. J. Wang, R. F. Miller and L. Huang, Clin. Chest Med., 2017, 38, 465-477.

15 G. D. Brown, D. W. Denning, N. A. Gow, S. M. Levitz, M. G. Netea and T. C. White, Sci. Transl. Med., 2012, 4, $165 \mathrm{rv113.}$

16 J. Berman and D. J. Krysan, Nat. Rev. Microbiol., 2020, 18, 319-331.

17 H. Hof, Drug Resist. Updates, 2008, 11, 25-31.

18 T. Weil, R. Santamaría, W. Lee, J. Rung, N. Tocci, D. Abbey, A. R. Bezerra, L. Carreto, G. R. Moura, M. Bayés, I. G. Gut, A. Csikasz-Nagy, D. Cavalieri, J. Berman and M. A. S. Santos, mSphere, 2017, 2, e00167.

19 L. E. Cowen and W. J. Steinbach, Eukaryotic Cell, 2008, 7, 747-764.

20 M. A. Pfaller, Am. J. Med., 2012, 125, S3-S13.

21 C. M. Martel, J. E. Parker, A. G. S. Warrilow, N. J. Rolley, S. L. Kelly and D. E. Kelly, Antimicrob. Agents Chemother., 2010, 54, 4920-4923.

22 A. Batta, B. S. Kalra and R. Khirasaria, J. Family Med Prim Care, 2020, 9, 105-114.

23 A. Chowdhary and A. Sharma, J. Global Antimicrob. Resist., 2020, 22, 175-176.

24 R. S. Shapiro, N. Robbins and L. E. Cowen, Microbiol. Mol. Biol. Rev., 2011, 75, 213-267.

25 D. J. Woods and T. M. Williams, Invertebr. Neurosci., 2007, 7, 245-250.

26 R. Laniado-Laborin and M. N. Cabrales-Vargas, Rev. Iberoam. Micol., 2009, 26, 223-227.

27 S. J. Kashyap, V. K. Garg, P. K. Sharma, N. Kumar, R. Dudhe and J. K. Gupta, Med. Chem. Res., 2012, 21, 2123-2132.

28 M. A. Ghannoum and L. B. Rice, Clin. Microbiol. Rev., 1999, 12, 501-517.

29 C. Biot, G. Glorian, L. A. Maciejewski and J. Brocard, J. Med. Chem., 1997, 40, 3715-3718.

30 G. Jaouen, A. Vessières and S. Top, Chem. Soc. Rev., 2015, 44, 8802-8817, and references therein.

31 N. P. E. Barry and P. J. Sadler, Chem. Commun., 2013, 49, 5106-5131.

32 M. Patra and G. Gasser, Nat. Rev. Chem., 2017, 1, 0066, and references therein.

33 Y. C. Ong, S. Roy, P. C. Andrews and G. Gasser, Chem. Rev., 2019, 119, 730-796.

34 G. Gasser and N. Metzler-Nolte, Curr. Opin. Chem. Biol., 2012, 16, 84-91.

35 C. G. Hartinger, N. Metzler-Nolte and P. J. Dyson, Organometallics, 2012, 31, 5677-5685.

36 I. Ott, Coord. Chem. Rev., 2009, 253, 1670-1681.

37 M. Patra, G. Gasser and N. Metzler-Nolte, Dalton Trans., 2012, 41, 6350-6358.
38 E. Boros, P. J. Dyson and G. Gasser, Chem, 2020, 6, 41-60.

39 R. Rubbiani, O. Blacque and G. Gasser, Dalton Trans., 2016, 45, 6619-6626.

40 E. W. Hunsaker, K. J. McAuliffe and K. J. Franz, J. Biol. Inorg. Chem., 2020, 25, 729-745.

41 A. Garoufis, S. K. Hadjikakou and N. Hadjiliadis, Coord. Chem. Rev., 2009, 253, 1384-1397.

42 N. V. Loginova, T. V. Koval'chuk, N. P. Osipovich, G. I. Polozov, V. L. Sorokin, A. A. Chernyavskaya and O. I. Shadyro, Polyhedron, 2008, 27, 985-991.

43 T. Mangamamba, M. C. Ganorkar and G. Swarnabala, Int. J. Inorg. Chem., 2014, 2014, 22.

44 M. R. Karekal, V. Biradar and M. Bennikallu Hire Mathada, Bioinorg. Chem. Appl., 2013, 2013, 16.

45 M. Kaya, E. Demir and H. Bekci, J. Enzyme Inhib. Med. Chem., 2013, 28, 885-893.

46 E. Pahontu, V. Fala, A. Gulea, D. Poirier, V. Tapcov and T. Rosu, Molecules, 2013, 18, 8812-8836.

47 E. Rodriguez-Fernandez, J. L. Manzano, J. J. Benito, R. Hermosa, E. Monte and J. J. Criado, J. Inorg. Biochem., 2005, 99, 1558-1572.

48 Z. H. Chohan, H. Pervez, K. M. Khan and C. T. Supuran, J. Enzyme Inhib. Med. Chem., 2005, 20, 81-88.

49 C. Biot, N. François, L. Maciejewski, J. Brocard and D. Poulain, Bioorg. Med. Chem. Lett., 2000, 10, 839-841.

50 Z. H. Chohan, Appl. Organomet. Chem., 2006, 20, 112-116.

51 G. Golbaghi, M.-C. Groleau, Y. López de los Santos, N. Doucet, E. Déziel and A. Castonguay, ChemBioChem, 2020, 21, 3112-3119, and references therein.

52 J. A. d. Azevedo-França, R. Granado, S. T. de Macedo Silva, G. d. Santos-Silva, S. Scapin, L. P. Borba-Santos, S. Rozental, W. de Souza, É. S. Martins-Duarte, E. Barrias, J. C. F. Rodrigues and M. Navarro, Antimicrob. Agents Chemother., 2020, 64, e01980.

53 N. L. Stevanović, I. Aleksic, J. Kljun, S. Skaro Bogojevic, A. Veselinovic, J. Nikodinovic-Runic, I. Turel, M. I. Djuran and B. Đ. Glišić, Pharmaceuticals, 2021, 14, 24.

54 T. Vago, G. Baldi, D. Colombo, M. Barbareschi, G. Norbiato, F. Dallegri and M. Bevilacqua, Antimicrob. Agents Chemother., 1994, 38, 2605.

55 K. Shalini, N. Kumar, S. Drabu and P. K. Sharma, Beilstein J. Org. Chem., 2011, 7, 668-677.

56 A. Stütz, Angew. Chem., Int. Ed. Engl., 1987, 26, 320-328.

57 G. Jaouen, A. Vessieres and S. Top, Chem. Soc. Rev., 2015, 44, 8802-8817.

58 D. Dive and C. Biot, Curr. Top. Med. Chem., 2014, 14, 1684-1692, and references therein.

59 S. Sansook, S. Hassell-Hart, C. Ocasio and J. Spencer, J. Organomet. Chem., 2019, 905, 121017.

60 X. Chai, J. Zhang, S. Yu, H. Hu, Y. Zou, Q. Zhao, Z. Dan, D. Zhang and Q. Wu, Bioorg. Med. Chem. Lett., 2009, 19, 1811-1814.

61 C. A. Ocasio, S. Sansook, R. Jones, J. M. Roberts, T. G. Scott, N. Tsoureas, P. Coxhead, M. Guille, G. J. Tizzard, S. J. Coles, H. Hochegger, J. E. Bradner and J. Spencer, Organometallics, 2017, 36, 3276-3283. 
62 A. Baramee, A. Coppin, M. Mortuaire, L. Pelinski, S. Tomavo and J. Brocard, Bioorg. Med. Chem., 2006, 14, 1294-1302.

63 N. C. Tice, S. Parkin and J. P. Selegue, J. Organomet. Chem., 2007, 692, 791-800.

64 H. Zhang, F. Xin, W. An, A. Hao, X. Wang, X. Zhao, Z. Liu and L. Sun, Colloids Surf., A, 2010, 363, 78-85.

65 R. S. Upadhayaya, S. Jain, N. Sinha, N. Kishore, R. Chandra and S. K. Arora, Eur. J. Med. Chem., 2004, 39, 579-592.

66 C. Sheng, W. Zhang, H. Ji, M. Zhang, Y. Song, H. Xu, J. Zhu, Z. Miao, Q. Jiang, J. Yao, Y. Zhou, J. Zhu and J. Lü, J. Med. Chem., 2006, 49, 2512-2525.

67 M. K. Lindvall and A. M. Koskinen, J. Org. Chem., 1999, 64, 4596-4606.

68 T. Bader, H.-U. Bichsel, B. GilomenImeld, I. Meyer-Wilmes and M. Sundermeier, Polymorphic forms of olopatadine hydrochloride and methods for producing olopatadine and salts thereof, US Pat., WO2007110761A8, 2007.

69 S. Keller, Y. C. Ong, Y. Lin, K. Cariou and G. Gasser, J. Organomet. Chem., 2020, 906, 121059.

70 M. Patra, T. Joshi, V. Pierroz, K. Ingram, M. Kaiser, S. Ferrari, B. Spingler, J. Keiser and G. Gasser, Chem. - Eur. J., 2013, 19, 14768-14772.

71 M. D. Hall, K. A. Telma, K. E. Chang, T. D. Lee, J. P. Madigan, J. R. Lloyd, I. S. Goldlust, J. D. Hoeschele and M. M. Gottesman, Cancer Res., 2014, 74, 3913-3922.

72 H. Sierotzki and G. Scalliet, Phytopathology, 2013, 103, 880-887. 73 H. Balba, J. Environ. Sci. Health, Part B, 2007, 42, 441-451.
74 L. Young, B. May, A. Pendlebury-Watt, J. Shearman, C. Elliott, M. S. Albury, T. Shiba, D. K. Inaoka, S. Harada, K. Kita and A. L. Moore, Biochim. Biophys. Acta, 2014, 1837, 1219-1225.

75 T. Niwa, T. Shiraga and A. Takagi, Biol. Pharm. Bull., 2005, 28, 1805-1808.

76 K. W. Henry, J. T. Nickels and T. D. Edlind, Antimicrob. Agents Chemother., 2000, 44, 2693-2700.

77 Y. Lee, E. Puumala, N. Robbins and L. E. Cowen, Chem. Rev., 2020, 121, 3390-3411.

78 H.-Y. Liu, V. Badarinarayana, D. C. Audino, J. Rappsilber, M. Mann and C. L. Denis, EMBO J., 1998, 17, 1096-1106.

79 Z. Liu and L. C. Myers, Antimicrob. Agents Chemother., 2017, 61, e01344-01317.

80 G. P. Moran, M. Z. Anderson, L. C. Myers and D. J. Sullivan, Curr. Genet., 2019, 65, 621-630.

81 J. L. Nishikawa, A. Boeszoermenyi, L. A. Vale-Silva, R. Torelli, B. Posteraro, Y.-J. Sohn, F. Ji, V. Gelev, D. Sanglard, M. Sanguinetti, R. I. Sadreyev, G. Mukherjee, J. Bhyravabhotla, S. J. Buhrlage, N. S. Gray, G. Wagner, A. M. Näär and H. Arthanari, Nature, 2016, 530, 485-489.

82 Y. Mahé, Y. Lemoine and K. Kuchler, J. Biol. Chem., 1996, 271, 25167-25172.

83 H. B. van den Hazel, H. Pichler, M. A. do Valle Matta, E. Leitner, A. Goffeau and G. Daum, J. Biol. Chem., 1999, 274, 1934-1941.

84 R. B. Ami, R. E. Lewis and D. P. Kontoyiannis, Clin. Infect. Dis., 2008, 47, 226-235. 